\title{
Más igualdad y otros modelos de varón, para erradicar la violencia e género
}

*Carmen Magallón Portolés,

Fecha de recibido: 14 de junio de 2011/ Aceptado: 17 de junio de 2011.

\section{RESUMEN}

C. Magallón señala que sin restar su particular importancia, no basta la creación, aprobación e incluso, la aplicación de las leyes punitivas ante la violencia intrafamiliar, a la par de ello, se debe continuar indagando los factores que existen y explican - en el sustrato profundo de la cultura patriarcal - la violencia de género contra la mujer; la asunción del problema por toda la sociedad y sus instituciones; cuestionar los universos conceptuales que legitiman la violencia, "el desarrollo de un concepto de igualdad entre los sexos, que no se detenga en la tradición masculina sino que englobe y tenga en cuenta la experiencia histórica de las mujeres y la puesta en circulación de nuevos modelos para el varón, capaces de romper la identificación entre masculinidad y violencia".

Palabras clave: Cultura patriarcal, violencia de género, nuevos universos conceptuales.

\section{ABSTRACT}

C. Magallon points out that is not enough the creation, approval and even, the implementation of laws against domestic violence, at the same time, the factors that exist and explain - from patriarchal culture- violence against women must continue investigating; acceptance of problem for all society and its institutions; to question conceptual universes that legitimize violence, "the development of a concept of equality between sexes that not only covers male tradition but also embraces and takes into consideration the women's historical experience and put in circulation new male models be able to break the identification between masculinity and violence.

Keywords: patriarchal culture, genre violence, new conceptual universes.

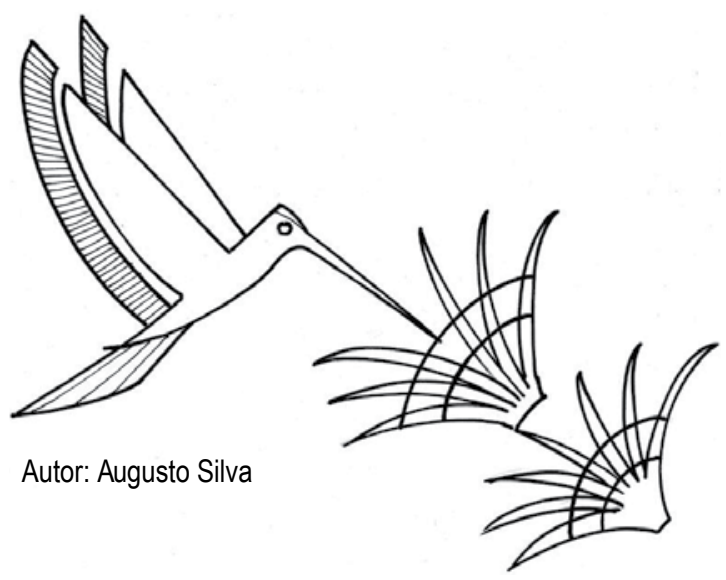

* Directora de la Fundación Seminario de Investigación para la Paz, Centro Pignatelli, Zaragoza www.seipaz.org , y miembro del grupo En pie de paz. 
$\mathrm{E}$ 1 debate sobre la violencia de género ha avanzado mucho en los últimos tiempos, un logro fundamental de la tenacidad y profundidad del pensamiento y la acción de los movimientos de mujeres, que han conseguido que el problema sea objeto de debate y de persecución, que haya pasado a la agenda política. Finalmente, también los medios de comunicación han contribuido dando visibilidad a una lacra que hasta hace unos años, se vivía como un asunto privado. Los casos graves de agresiones, de muertes de mujeres a manos del novio, del marido, o del compañero, han saltado a las pantallas y los diarios. Estos casos más graves son la punta del iceberg de un conjunto más amplio de ataques, menos llamativos, que conforman el universo conocido como violencia de género, un tipo de violencia en el que, de un modo estadísticamente significativo, abrumador podría decirse, el agresor es un hombre y la víctima una mujer. ${ }^{1}$

\section{¿Puede hacer algo la educación} al respecto, o debemos atenernos solamente $-y$ no es poco- al castigo de los culpables y la protección de las victimas?

Hay que decir que proteger a las víctimas y castigar a los culpables es una actuación básica dentro de un Estado de derecho, que tiene la obligación de proteger la integridad de sus ciudadanos y ciudadanas. Un terreno en el que todavía queda mucho por mejorar. Por ejemplo, no parece muy claro que sacar a una mujer maltratada de su casa, una de las actuaciones más frecuentes cuando se denuncia una agresión, sea el tipo de acción más justa. Es cierto que se hace para proteger a la víctima, pero a la vez esta medida está suponiendo un castigo para la mujer: mientras el agresor permanece en su residencia y puede continuar su vida, ella tiene que desarraigarse y hasta incluso esconderse, como si fuera la culpable. Por eso, sacar a la mujer de su casa tal vez sea una actuación adecuada pero desde luego no es justa.

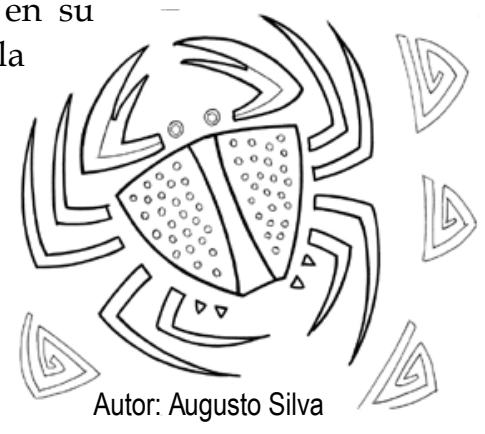

Este desfase entre adecuación y justicia, de una actuación preventiva, es también, en cierta medida, revelador. Refleja el predominio de una mentalidad que tiene raíces profundas. Y es en estas raíces en las que la educación, tanto en su sentido amplio, como en su sentido restringido, sigue teniendo una influencia decisiva. Sobre todo la educación como socialización, la que actúa por medio de la transmisión de valores en la familia, entre los pares, a través de los medios de comunicación, en la estructura social. También la que se transmite en la escuela. Quienes trabajamos hacia una cultura de paz, que ya hoy tratamos de darle vida, asumimos que ni el ser humano mujer ni el ser humano hombre, están determinados biológicamente. Pese a la dificultad de desentrañar qué parte de nosotros corresponde a lo natural biológico y qué parte a la socialización, el modelo que admite la interacción recursiva entre una base biológica y el papel activo de la socialización, parece el más adecuado para dar cuenta del amplio abanico de comportamientos de unos y otras.

Propongo que ese esfuerzo educativo hacia el cambio de mentalidad vigente vaya encaminado a revisar los modelos sociales que ejercen de medida en la conformación de nuestro ser mujeres y hombres. Estos modelos se refuerzan por la relación que se crea entre ambos sexos. Basada inicialmente en el amor, algo no funciona en esta relación cuando tan a menudo se muestra destructiva. Y averiguar qué es compete a la sociedad en su conjunto. De ahí que llamar educativo a este esfuerzo no tenga tanto el sentido de acotarlo en determinados ámbitos cuánto dotarlo de contenido transformador.

\section{¿Hacia donde habríamos de orientar los esfuerzos educativos para avanzar en la transformación profunda del actual estado de cosas?}

Como punto de partida ineludible para avanzar, se impone la profundización en la coherencia social de asumir que la violencia de género no es un asunto privado que concierna solamente a la relación de pareja,

1 Este texto fue presentado en el I Congreso Hispanoamericano sobre Educación y Cultura de Paz, celebrado en Granada, España. Una versión ampliada de este mismo tema puede verse en MAGALLÓN (2003). 
sino que se trata de un problema que compete a todos, en el que toda la sociedad y todas las instituciones han de implicarse. En esta asunción social se está avanzando como nunca antes pero todavía hay un camino por recorrer.

En cuanto a ir más allá, el esfuerzo de profundización nos lleva a indagar qué fuerza es

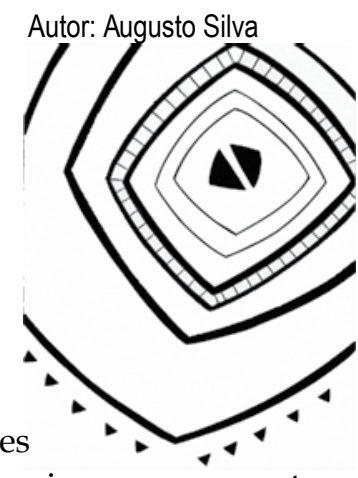
la que impulsa a los hombres a ejercer la violencia, llegando incluso matar a las mujeres, en un gran número de casos aún sabiendo que la ley les hará pagar por su crimen. Se necesita analizar la realidad compleja en la que se asientan estas conductas, perseguir las raíces de su conformación, proponer teorías explicativas y también proponer conceptualizaciones alternativas, visiones nuevas que puedan abrir el horizonte de opciones para ser hombres y mujeres, en una sociedad en cambio.

Las ciencias, tanto sociales como naturales, han jugado -y juegan- un papel importante en la legitimación de determinados prejuicios acerca de los sexos y de otras diferencias. Entre otras cuestiones, han interpretado el significado del sexo, el significado del color de la piel y el significado de las culturas. Las teorías biológicas, psicológicas y de la medicina, han influido de un modo muy importante en la conceptualización de qué es ser un hombre y una mujer. Y con sus interpretaciones han establecido límites para las aspiraciones de unas y otros, potenciando o negando el ejercicio de la libertad de los hombres y mujeres de carne y hueso.

La comunidad científica formada a lo largo de los siglos por una mayoría de hombres, elaboró teorías altamente sesgadas sobre la naturaleza de la mujer -y del hombre, pensado como genérico humano-. Estos sesgos, alimentados por los prejuicios sociales de la época, sirvieron de legitimación de los mismos prejuicios que estaban en su origen. Pasividad o agresividad, sentimiento o razón, debilidad o fuerza, fueron atributos que se atribuyeron a unas y otros, constriñendo los modelos sociales y encasillando el desarrollo personal y social de ambos sexos.

También sabemos, al menos desde Kuhn, que cuando cambia un paradigma explicativo, el mundo mismo cambia con él. Que el sentido de vivir en un mundo diferente se abre con la novedad de la perspectiva, con la emergencia de una nueva forma de ver las cosas. De la nueva visión surgen nuevos hechos y la realidad se amplía. Pero para que esto ocurra hay que atreverse a poner en cuestión un universo conceptual dado, cosa que suelen hacer los grupos humanos que tienen algo que ganar en el cambio. No es casualidad que la crítica al conocimiento establecido haya surgido de grupos que no fueron bien tratados por la corriente principal de ese saber los grupos del tercer mundo y los grupos que defienden la naturaleza.

En esta línea de razonamiento, la siguiente pregunta que se abre es qué universo conceptual hay que cuestionar si queremos avanzar en la erradicación de la violencia contra las mujeres. Aquí es donde se sitúa mi propuesta, cuya novedad no es tanto el contenido sino el énfasis en relacionarlo con el problema de la violencia de género.

Pienso que, con objeto de remover universos conceptuales estancados, habría que dar nuevos impulsos educativos e institucionales a dos líneas de pensamiento-acción:

Una, el desarrollo de un concepto de igualdad entre los sexos que no se detenga en la tradición masculina sino que englobe y tenga en cuenta la experiencia histórica de las mujeres; y dos, la puesta en circulación de nuevos modelos para el varón capaces de romper con la identificación entre masculinidad y violencia.

\section{Una igualdad que conceda universalidad a la experiencia histórica femenina}

Pensadoras y pensadores que han analizado el fenómeno de la violencia contra las mujeres están de acuerdo en que es el reflejo de una desigualdad de poder y autoridad, que está arraigada en la cultura profunda, y que se transmite por múltiples vías en la socialización de unas y otros. La identidad del varón crece entrelazada con esa posición de dominación que ejerce de modelado y que puede llegar a hacerle sentir como amenaza la afirmación de la mujer como ser libre. Mediante el maltrato, el varón trata de dominar y doblegar a la mujer, controlando su vida y su voluntad, y llegando al extremo de eliminarla físicamente cuando no consigue lo que se propone. Este ejercicio cotidiano de poder está mostrando una mentalidad discriminatoria que da pie a una relación 
viciada. El varón que maltrata no reconoce en la mujer a un ser humano igual.

Pese a que la igualdad formal de hombres y mujeres ante la ley es en nuestra sociedad un hecho y un derecho inalienables, estamos comprobando, en el día a día, cómo el reconocimiento formal no lleva consigo su puesta en práctica real. Las leyes son necesarias para regular la convivencia y también actúan sobre las conductas pero, en un momento histórico dado, no son sólo las leyes las que acotan la acción humana. El peso del pasado es demasiado poderoso para ser eliminado de un día para otro. En particular, el lugar simbólico de la mujer y el hombre arraiga en capas profundas de la cultura que nos conforma y que han sido y siguen siendo alimentadas por las creencias, la literatura, el arte, la filosofía, la ciencia, los relatos históricos, las costumbres y tradiciones. Al tratarse de comportamientos y modelos arraigados en la mentalidad profunda, su desvelamiento y superación necesita un debate social y cultural más amplio. El enunciado de leyes de igualdad entre los sexos, siendo un paso fundamental y necesario, no es suficiente.

Por eso, pese a lo que pudiera parecer, hacer hincapié en la igualdad es una tarea que todavía nos concierne. Y sobre la que hay que seguir debatiendo pues no existe un acuerdo generalizado acerca de lo que esta igualdad ha de significar en la práctica. A algunas y algunos nos preocupa que los materiales para construir esa igualdad provengan fundamentalmente de los viejos esquemas masculinos, basados en la tradición del hombre blanco, occidental y de clase media. Ese concepto de igualdad sigue dejando en la invisibilidad la diversidad y la autoridad de la experiencia femenina histórica, y se muestra incapaz de desarraigar la desconsideración social hacia las mujeres, embebida en las capas profundas de la cultura.

La desigualdad se plasma también en cómo se conciben y estructuran las instituciones, construidas y definidas a la medida del varón arquetípico antes mencionado. Podemos preguntarnos si la presencia femenina se ha hecho significativa en la esfera pública, si ahora existimos más, y por qué, si se ha percibido una corriente de aire fresco con la llegada de las mujeres a algunos de los espacios públicos en los que antes estaban solos los varones, o si han sido absorbidas en el modelo dominante que las vuelve invisibles. Preguntarnos si el hecho de que siga habiendo tan pocas mujeres en los puestos clave de decisión política, de la economía, de la tecnociencia, no está hurtando a la sociedad la parte de autoridad femenina que podría servir de modelo para erradicar esos patrones de desigualdad profunda. Preguntarnos por el lugar real que ocupa el legado de las mujeres en la esfera pública, incluyendo los propios hábitos de ser y de razonar de los hombres del siglo XXI.

Lo anterior nos lleva a la necesidad de reequilibrar la asimetría simbólica del peso del legado masculino y femenino en la cultura. Hemos universalizado una parte, tampoco toda, de la experiencia masculina, y dejado en la sombra otras contribuciones, de hombres y mujeres, lamentablemente aquellas más ligadas al cuidado y la convivencia pacíficas. El poder de la fuerza y la violencia impregnan el legado humano históricamente transmitido, hasta hacerse indistinguibles, mientras queda pendiente universalizar la experiencia femenina. Menos cooptadas por la socialización para el ejercicio de la fuerza, las mujeres han recreado el cuidado de las personas como una misión importante de mantenimiento de la especie, y aún tratándose de una experiencia plural y desigual, su experiencia es un legado civilizatorio que es preciso tener en cuenta para la construcción de la igualdad.

¿Cómo puede erradicarse el desprecio por las mujeres si no se concede valor social a lo que han hecho en el pasado y a lo que hacen en el presente? Si queremos una verdadera igualdad entre hombres y mujeres, habrá que poner en plano de igualdad la experiencia histórica de ambos sexos y utilizar de algún modo los ladrillos -la experiencia- que ellas acumularon a lo largo de la historia, para la construcción del legado universal del conjunto humano.

Siempre he pensado que sacar a la luz los logros y experiencia de las mujeres, por ejemplo sus aportaciones a la ciencia (MAGALLÓN, 1998), contribuye a la construcción de una cultura de paz; que la emergencia social de la sabiduría de las mujeres, menos conocida y reconocida que la de los varones, 
puede colaborar a un cambio, aportando significados nuevos a la cultura y proporcionando raíces para una igualdad más profunda, capaz de ayudar a la erradicación de la violencia de género.

En la insistencia por alcanzar una auténtica igualdad quisiera añadir un matiz, y es que ésta no puede darse, sin un reconocimiento de autoridad en el otro. La igualdad a que hago referencia supone el reconocimiento de la mujer como miembro de la comunidad, de pleno derecho, con todo lo que eso significa, incluida su diferencia. Esto tiene que ver con la puesta en cuestión de que el varón sea la medida de la igualdad, la norma. Una norma que emana de una sola parte, en este caso de uno de los sexos, siendo parcial se totaliza y, de este modo, anula a la otra parte. La norma común ha de construirse desde la doble experiencia y ha de modificar a ambos sexos. No hay reconocimiento de autoridad si no se hace visible la diversidad y los logros históricos de las mujeres en los lugares culturales donde éstos se dieron. En el cuidado de los demás, sí, pero también en la ciencia, en el pensamiento, en el arte, en todo resquicio de la cultura convivida. Aunque las mujeres se hayan abierto paso en las profesiones, en las instituciones, en la medida en que su presencia no modifique la norma de igualdad, modificando también al varón, siguen sin tener un lugar de referencia simbólica.

El recorrido civilizatorio de las mujeres, con sus aportaciones a los campos específicos de la cultura y -a la vez- el trabajo de cuidado como central, aporta aspectos sustanciales para una noción de humanidad más evolucionada y civilizada. Es este modelo de compatibilización el que cobra importancia para una cultura de paz, una cultura en la que la vida humana, y no los intereses económicos; el cuidado del otro -y no la agresión y la dominación- estén en el centro.

Para este cambio no podemos quedarnos con una visión de las mujeres como víctimas. La imagen de las mujeres como víctimas es paralizante y no hace justicia a la diversidad, riqueza, empuje y protagonismo de los grupos de mujeres que tratan de mejorar el mundo en el que vivimos. Incluso en el maltrato, las mujeres no sólo son víctimas. Las mujeres hace tiempo que más que pedir, estamos ofreciendo. Hace tiempo que sin negar a las víctimas, nos hemos situado más allá del paradigma de la víctima. Las mujeres son protagonistas de la supervivencia en muchas partes del planeta. Defendiendo la calidad de la educación, o de los alimentos, los servicios en los barrios, la capa de ozono o el mantenimiento de los bosques. Esta sabiduría para la supervivencia es la que se ha expresado también en otros campos de la cultura, a lo largo de la historia. Y es la que ha de ponerse en circulación social para contribuir a la socialización de ambos sexos.

\section{Nuevos modelos de varón}

Los informes que dan los vecinos y compañeros de los maltratadores, esos hombres que en muchos casos llegan a asesinar a la mujer con la que convivían, suelen coincidir en afirmar que se trataba de un vecino normal. En diagnósticos posteriores, los psicólogos y psiquiatras suelen confirmar esa aseveración. Es decir, la patología de esta conducta, de maltratar e incluso asesinar a mujeres, se da en hombres normales, lo que induce a pensar que es la normalidad la que incorpora una distorsión, y que por tanto ha de ser revisada. Puede parecer contradictoria la afirmación de una conducta patológica que se da en hombres normales. No lo es si tomamos normalidad no por su contenido sino como patrón de comportamiento extendido. Para las mujeres maltratadas, y es una percepción a generalizar, esta normalidad no tiene nada de normal (LORENTE, 2001).

Los estudios al respecto, realizados desde campos como la psicología, la sociología y la antropología, permiten pensar que son las relaciones sociales -materiales y simbólicas- que se dan entre hombres y mujeres, apoyadas por una concepción determinada de lo que significa ser un hombre y una mujer (modelos de identidad), las que están en la base de esta distorsión que conduce a la violencia. Unas relaciones marcadas por la desigualdad y la discriminación históricas, que han conformado unos modelos de identidad determinados.

La perspectiva de la desigualdad se ha planteado más arriba. La de las identidades voy a enfocarla centrándome en el agresor pues el modelo de mujer, sumisa y pasiva, 
que conformaría la identidad de la víctima, de la mujer, está ya puesto en cuestión desde hace tiempo por el pensamiento feminista. La crítica al esterotipo de mujer que se adecúa a la relación de maltrato existe en el movimiento feminista desde hace décadas $\mathrm{y}$, de hecho, al hilo de la misma emergieron y existen nuevos modelos de mujer en circulación. Es en

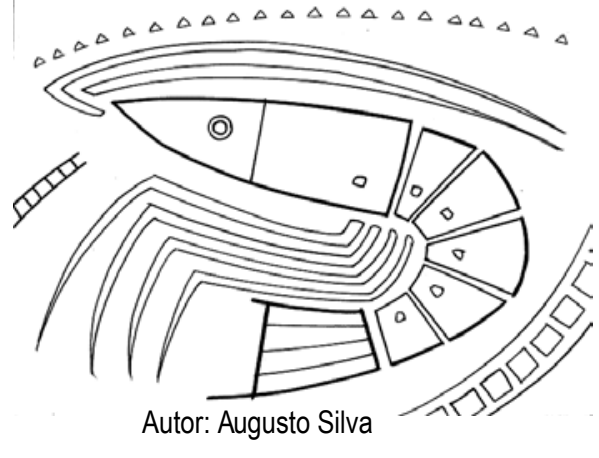
el caso de los varones, en el que la crítica análoga está por llegar o se hace sólo desde los márgenes. En este caso, los arquetipos que siguen teniendo un gran peso como modelo, son los que identifican masculinidad con fuerza y dominio, y llegado el caso con el ejercicio de la violencia. A partir de aquí, y de un modo metafórico, nos preguntamos si la violencia contra las mujeres no será el resultado de una guerra de identidad que concierne a los hombres.

El modelo de varón con el que, en última instancia, se sigue midiendo un hombre en momentos duros de su vida, el modelo que en momentos de crisis parece regir, es un arquetipo en el que la dureza, el éxito, el ocultamiento de los sentimientos y la competitividad extrema que condicionan a valorar por encima de todo la victoria y la gloria, y a encerrarse en las dicotomías nosotros/ellos o ganar/perder -el ejercicio de la dominación-, forman parte del núcleo duro de su identidad. Es aquel que niega en sí cualquier rasgo femenino; en el que se da la ausencia de capacidades empáticas, de ponerse en el lugar del otro. Se trata de un arquetipo que hace sentirse profundamente herido y humillado al varón ante la libertad de una mujer diciendo no, o marchándose. Es este modelo el que todavía sigue siendo sutil o brutalmente hegemónico, en la socialización de los niños. Aunque existe una evolución de este arquetipo que deviene en diversidad en muchas capas de la población, todavía ellos desde la infancia, tienen que soportar una variedad de presiones sociales, que les empujan a construir y demostrar su masculinidad a través de conductas adecuadas al modelo.

De ahí el destacar la importancia que posee, para la eliminación de las raíces profundas de la violencia contra las mujeres, la persistencia en la reflexión y el rechazo social de los aspectos más nefastos de ese estereotipo masculino, aquellos que atan a los varones a los comportamientos violentos, en nombre de su rol. Y al mismo tiempo, a la vez que se rechaza el viejo modelo, la oferta de nuevos modelos de identificación para el varón, que favorezcan el cambio de los hombres. Esos modelos también existen ya, porque el mundo es grande y diverso, pero todavía no han desplazado al varón dominante de su puesto hegemónico. Hoy, lo estamos viviendo, muchos hombres de nuestro entorno son sensibles, empáticos, cuidadores y no especialmente agresivos; pero el modelo al que dan vida tiene una menor visibilidad y hegemonía social (en la toma de decisiones políticas, en el cine, en la $\mathrm{TV}$, en las novelas), de modo que nuestros adolescentes siguen alimentándose con los grandes mitos de la fuerza y la dominación masculina. Los nuevos modelos han de hacerse visibles y significativos, para que trasciendan culturalmente.

¿Qué papel tienen en este cambio las mujeres? Pues hay que decir que son muchas las que siguen reafirmando esa forma de masculinidad, cercana al mito y al estereotipo dominador, sobre todo con sus elecciones amorosas. No obstante, y en coherencia con la valoración social de quién es importante son fundamentalmente los varones los que avalan con su aplauso la masculinidad. Quienes hemos trabajado con adolescentes sabemos cómo pesa en su comportamiento la opinión y la mirada de sus compañeros, varones. Difícilmente cuajará una crítica del rol y valores masculinos que no sea avalada por el grupo de iguales. Se trata, pues, de una tarea social que han de tomar en sus manos los propios hombres.

Ya se ha mencionado como las mujeres, como grupo subordinado, pusieron en cuestión la identidad y roles que les fueron asignados. La conceptualización de género, potenciada desde el feminismo, trajo consigo un cuestionamiento de la construcción social de la mujer. Y el feminismo supo aprovecharla. De modo análogo a cómo cuestiona la construcción social de la mujer, la noción de género permite también cuestionar la construcción social del varón. Pues parafraseando a Simone de Beauvoir, podemos decir que no se nace varón sino que se llega a serlo.

Desde el feminismo, es un clamor que los hombres tienen que proceder a deconstruir la masculinidad, del mismo 
modo que las mujeres llevamos años haciendo lo propio con la femineidad. Ambas, masculinidad y feminidad son expresiones del ser hombre o mujer modificables. A hacer real y profundo el cambio iniciado por las mujeres pueden contribuir -es necesario que contribuyan- de un modo fundamental los movimientos de autocrítica surgidos en el seno de los propios hombres, que han decidido salir del silencio y asumir un debate acerca del rol que la cultura les asigna y del modelo de masculinidad dominante. La forma actual de la masculinidad no ha sido siempre así, es modificable y hay que buscar las vías para hacerlo.

Creo, junto a muchas otras personas, que es el momento de que los hombres que toman en serio el problema de la violencia contra las mujeres, lo sientan como propio y se organicen en torno a él. Como primer paso, tienen pendiente debatir sobre sí mismos, constituirse en objeto de debate (sujeto-objeto), y ofrecer de este modo -modélico- una vía de crítica del estereotipo masculino dominador y violento, a los niños y adolescentes.

Algunos hombres cuando oyen todo lo anterior se sienten ofendidos, piensan que se les está denigrando. Pero lo que se denigra es el sistema patriarcal. Junto a la crítica hay que reseñar muchos aspectos positivos en el modelo y rol masculinos. El cambio que se propone no afecta al hombre por ser hombre, sino al modelo, y en aquellos aspectos de la masculinidad que ligan ser hombre con ejercicio de la violencia. Algunos sienten sentimientos de culpa. Pero los sentimientos de culpa no ayudan a salir del problema. La mayoría de los hombres sencillamente están atrapados en este sistema, como lo están las mujeres. Y muchas mujeres son más responsables de la perpetuación de este sistema que muchos otros hombres, sobre todo las que usan los prejuicios partriarcales en su propio beneficio.

En este tema es esperanzador observar el nacimiento de un movimiento de hombres que rechazan el sistema productor de estas consecuencias, es decir, el patriarcado. En los últimos años han crecido los grupos que ponen en cuestión las nociones hegemónicas de la masculinidad. Movimientos que arrancan de la necesidad de problematizar uno de los dos polos de la caracterización de género, que estos estudios han destronado de su universalidad. Poner el acento en los hombres puede ayudar a deshacer el nudo que hace equivaler humanidad a hombres. Hemos hablado, hablamos mucho de mujeres, y tal vez sea el momento de hablar de los hombres; no como el paradigma de universalidad, sino precisamente en su parcialidad, como uno de los dos sexos.

\section{¿Qué interés pueden tener los hombres en adoptar compromisos hacia el cambio de su rol?}

Algunos autores, que llevan tiempo con estas reflexiones, apuntan lo que también podemos observar a nuestro alrededor, si participamos en la socialización de niños y niñas. Que son muchos los niños y adolescentes varones que están sufriendo, en el proceso de adecuarse al modelo de varón hegemónico. Y que como ha escrito Michael Kaufman (2000), canadiense que inició la campaña del lazo blanco contra la violencia hacia las mujeres, las inseguridades personales conferidas por la incapacidad de pasar la prueba de hombría, o simplemente por la amenaza del fracaso, son suficientes para llevar a muchos hombres a un torbellino de miedo, aislamiento, ira, autocastigo, autorrepudio y agresión. En dicho estado emocional, la violencia se convierte en un mecanismo compensatorio. El mismo autor señala que las formas a través de las que los hombres adultos ejercen el poder social e individual son fuente de un enorme temor, aislamiento y dolor para sí mismos. Las exigencias tan elevadas que exige el estereotipo se presentan como incumplibles, pero a la vez su incumplimiento o transgresión es causa de angustia, al ser interpretado como duda de si se es o no un hombre. Algunos se preguntan si los roles masculinos están dañando a los hombres, algo que es patente para Luis Bonino (2000) que habla de malestares y molestares, en torno al ser hombre. Desde el interés por indagar las raíces que sustentan el ejercicio de la violencia en la construcción del varón, han surgido grupos, seminarios y centros de estudio de la masculinidad. Los hay en España y en muchos países del mundo. Por señalar algunos, existen grupos de hombres contra la violencia en Nicaragua, en Canadá, en Estados Unidos. Hay también iniciativas que tratan de colocar el tema en la agenda política internacional.

En los países nórdicos, con el apoyo político y económico del Consejo Nórdico de los Ministros, UNESCO, UNICEF y el Consejo de Europa, en 1997 se llevaron a cabo un total de 6 conferencias nórdicas e internacionales sobre roles y valores masculinos. Desde entonces están trabajando por llevar a cabo una Conferencia Mundial de Naciones 
Unidas sobre este tema. La idea fue lanzada inicialmente por Eva Moberg, columnista de uno de los diarios suecos de mayor tirada, Dagens Nyheter, en una reunión de la Red de Mujeres Nórdicas por la paz, que hizo suya la idea. La realización de esta Conferencia ha sido defendida en diversos foros internacionales, sobre todo por hombres y mujeres de los países nórdicos, también a través de la Cruz Roja y la Luna Roja Internacionales, en Ginebra, en 1995 y 1999. Tal conferencia puede ser considerada análoga a las Conferencias de Naciones Unidas sobre las Mujeres que se han venido desarrollando en 1975, 1980, 1985 y 1995. Todas fueron importantes para el avance de la situación de las mujeres en el mundo. De modo análogo, el llevar a cabo una sobre roles y valores masculinos podría ser un impulso crucial en el avance de nuevos modelos para los varones y, desde nuestro análisis, para la erradicación de la violencia de género. $\mathrm{Si}$, como afirman algunas analistas, todos los problemas que fueron tratados en las Conferencias de Naciones Unidas sobre las Mujeres, tienen sus raíces en el rol de los hombres y en la cultura masculina, el cuestionamiento de estos tópicos en un escenario internacional pasa a ser una tarea urgente.

\section{BIBLIOGRAFÍA}

BADINTER, Elisabeth (1986) L'un est l'autre, Paris, Odile Jacob.

BADINTER, Elisabeth (1993) XY. La identidad masculina, Madrid, Alianza.

BARNET, Richard (1973) Roots of war: The Men and Institutions Behind U.S. Foreign Policy, Nueva York, Penguin Books.

BONINO, Luis (2000) Varones, género y salud mental: deconstruyendo la "normalidad" masculina, en Marta Segarra y Angels Carabi (eds.) Nuevas masculinidades, Barcelona, Icaria.

BUTLER, Judith (1990) Gender Trouble. Feminism and the Subversion of Identity, New York and London, Routledge.

CHODOROW, Nancy (1978) El ejercicio de la maternidad. Psicoanálisis y sociología de la maternidad y paternidad en la crianza de los hijos, Barcelona, Gedisa, 1984.

CLATTERBOUGH, Kenneth (1990) Contemporary Perspectives on Masculinity. Men, Women and Politics in modern Society, Boulder, Westiew Press.

DARCY DE OLIVEIRA, Rosiska (1992) Elogio da diferença.
O feminino emergente, São Paulo, editora brasiliense, $2^{\mathrm{a}} \mathrm{ed}$.

ELSHTAIN, Jean B. (1995) Women and War. Chicago, The University of Chicago Press.

FAUSTO STERLING, Anne (1985) Myths of Gender. Biological Theories about Women and Men, New York, Basic Books.

FISAS, Vicenç (ed.) (1998) El sexo de la violencia. Género y cultura de la violencia, Barcelona, Icaria.

GALTUNG, Johan (1996) Peace by peaceful means. Peace and Conflict, Development and Civilization, London, Sage Publications.

GILLIGAN, Carol (1982) In a Different Voice. Psychological Theory and Women's Development, Cambridge, Harvard University Press.

GILMORE, David (1990) Manhood in the Making. Cultural Concepts of Masculinity, Yale University Press.

KAUFMAN, Michael (1997) Las experiencias contradictorias del poder entre los hombres, en Teresa Valdés y José Olavarría (eds.) Masculinidad/es: Poder y crisis, Santiago de Chile, Isis Internacional, 63-81.

LORENTE ACOSTA, Miguel (2001) Mi marido me pega lo normal. Agresión a la mujer: realidades y mitos, Barcelona, Ares y Mares.

MAGALLÓN PORTOLÉS, Carmen (1998) Pioneras españolas en las ciencias. Las mujeres del Instituto Nacional de Física y Química, Madrid, CSIC, Estudios sobre la ciencia, $\mathrm{n}^{\circ} 24$.

MAGALLÓN PORTOLÉS, Carmen (2001) El pensamiento maternal. Una epistemología feminista para una cultura de paz, en Francisco A. Muñoz (ed.) La paz imperfecta, Granada, Universidad de Granada, 123-141.

MAGALLÓN PORTOLÉS, Carmen (2003) Compartir el cuidado, compartir la autoridad: hacia una cultura del respeto entre hombres y mujeres, en Fundación Seminario de Investigación para la Paz (ed.) Pacificar violencias cotidianas, Zaragoza, Gobierno de Aragón, en prensa.

MARTÍNEZ GUZMÁN, Vicent (2001) Filosofía para hacer las paces, Barcelona, Icaria.

MIEDZIAN, Myriam (1991) Chicos son, hombres serán. Cómo romper los lazos entre masculinidad y violencia, Madrid, horas y Horas, 1995.

MONTOYA TELLERÍA, Oswaldo (1998) Nadando contra corriente. Buscando pistas para prevenir la violencia masculina en las relaciones de pareja, Managua, Puntos de Encuentro. 